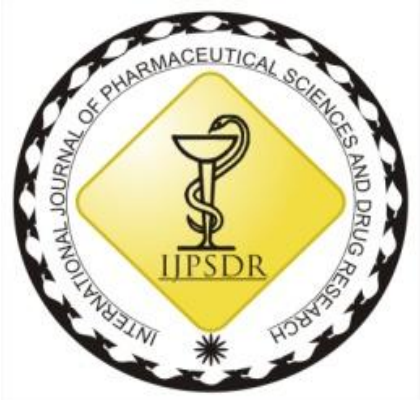

ISSN: 0975-248X

RESEARCH ARTICLE CODEN (USA): IJPSPP $(\mathrm{cc})$ EY-NG-SA

\title{
Biogenic Synthesis of Silver Nanoparticles (AgNPs) using Celosia cristata L. Leaves Extract and Their Antimicrobial Activity against Otorhinolaryngological Isolated Pathogen
}

\author{
Ramveer Singh*, Navneet Kumar \\ Department of Botany and Microbiology, Gurukula Kangri Vishwavidyalaya, Haridwar-249404, Uttarakhand, India
}

Copyright (C) 2019 Ramveer Singh et al. This is an open access article distributed under the terms of the Creative Commons AttributionNonCommercial-ShareAlike 4.0 International License which allows others to remix, tweak, and build upon the work non-commercially, as long as the author is credited and the new creations are licensed under the identical terms.

\section{ABSTRACT}

This work focused on the biogenic synthesis of silver nanoparticles (AgNPs) by silver nitrate using Celosia cristata leaves extract in four different solvents namely petroleum ether, acetone, methanol, and water. Silver nitrate and leaves extract were used as a precursor and capping reducing agent respectively. Biogenic AgNPs were characterized and identified by UV-Vis spectrophotometer, X-ray diffraction (XRD), Field emission scanning electron microscope (FE-SEM), and energy dispersive spectroscopy (EDX). Disc diffusion method was used for antibacterial activity of AgNPs and effective antibacterial activity was shown against IS-3, IS-4, IS-6, and IS-7 as compared to a positive control (ciprofloxacin).

Keywords: Silver nanoparticles, FE-SEM, X-ray diffraction, antibacterial activity, Celosia cristata.

*Corresponding author: Mr. Ramveer Singh

Address: Department of Botany and Microbiology, Gurukula Kangri Vishwavidyalaya, Haridwar-249404, Uttarakhand, India

E-mail $\bowtie$ : ramveer.gangwar@outlook.com

Relevant conflicts of interest/financial disclosures: The authors declare that the research was conducted in the absence of any commercial or financial relationships that could be construed as a potential conflict of interest.

Received: 19 September, 2019; Revised: 24 October, 2019; Accepted: 31 October, 2019; Published: 30 November, 2019

\section{INTRODUCTION}

From the last few decades, the field of nanotechnology had gained much importance in several research and development working areas. Silver nanoparticles (AgNPs) are universally utilized metal nanoparticles [1], these nanoparticles were also used in personal care, household products and clothes. [2-3] Synthesis of AgNPs can be done by radiation, chemical, photochemical and electrochemical methods. However, these processes are very slow, costly and hazardous to humans. Biogenic synthesized AgNPs are good alternatives to the above methods. [4] Biogenic synthesized AgNPs are nontoxic to human and effectively works against bacteria at very low concentration. [5] Many bacteria developed antibiotic resistance to most of the antibiotics, so there is a requirement of alternate antimicrobial substances. AgNPs works against all pathogenic bacteria and no bacteria developed antimicrobial resistance against it. [6] C. cristata is a household decorative plant belongs to family Amaranthaceae. It is commonly known as Fire flame and Cockscomb in English and Chi Kuan in Chinese. This is a herbaceous plant with a height of up to $30 \mathrm{~cm}$. Leaves are simple and alternate with green sometimes red or purple in color. Flowers are divided into three parts i.e. spikes, plumes, and crests. Flowers 
also vary in color; red, yellow, orange, and pink are common. This plant showed the presence of flavonoids, alkaloids, carbohydrate, gum, protein, steroids, saponins, amino acids, vitamin B1, B2, C and E. C. cristata used as traditional medicine for the treatment of uterine bleeding, hemorrhoids, bloody stool, wound healing, burning, leucorrhoea, amenorrhea, hyperactivity of liver and dysentery. ${ }^{[7-9]}$

This research paper focused on the biosynthesis of AgNPs by different solvents (petroleum ether, acetone, methanol, and water) leaves extract of $C$. cristata which were characterized by UV-VIS spectrophotometer, XRD, EDX, FE-SEM, and their antimicrobial activity was also investigated.

\section{MATERIALS AND METHODS}

\section{Experimental}

Silver nitrate $\left(\mathrm{AgNO}_{3}\right)$ used as a precursor for $\mathrm{AgNPs}$ synthesis was purchased from Sigma-Aldrich. Sterile cotton swab, Muller Hilton agar medium, nutrient agar medium, nutrient broth, and antimicrobial susceptibility discs were purchased from HiMedia.

\section{Plant Material}

These plants were collected from Sitarganj, Uttarakhand and identified as C. cristata by the BSI Dehradun and herbarium sheet with the Acc. No. 118058 was submitted.

\section{C. cristata leaves extract preparation}

Young green leaves of the plants were taken and washed with running water and shade dried at room temperature for 15-16 days. $200 \mathrm{~g}$ of powdered leaves were taken for successive solvent extraction by the Soxhlet apparatus.

\section{Isolation of otorhinolaryngological pathogen}

All otorhinolaryngological specimens were collected by sterile cotton swab from the otorhinolaryngological infection suffering patients. All specimens were cultured on nutrient agar medium at $37^{\circ} \mathrm{C}$ for 24 hours and all isolated were stored on nutrient agar medium slants at $4^{\circ} \mathrm{C}$ for further use.

\section{Silver nanoparticles (AgNPs) synthesis}

For the AgNPs synthesis, $10^{-3}$ molar $\left(10^{-3} \mathrm{M}\right)$ and $10 \%$ $(\mathrm{w} / \mathrm{v})$ silver nitrate solution leave extract were prepared. $10 \mathrm{ml}$ of leaves extract was added in $90 \mathrm{ml}$ of $10^{-3} \mathrm{M} \mathrm{AgNO}_{3}$ solution. After adding the leaves extract in the solution, the color change in the solution occurred slowly. The formation of silver nanoparticles was confirmed by the brown color of the solution. This solution was left in dark for 24 hours. After 24 hours centrifugation of colloidal suspension was done for 20 minutes at $10000 \mathrm{rpm}$. Unbound particles were removed by centrifugation and supernatant was replaced by double distilled water. Settled nanoparticles were dried in the oven at $55^{\circ} \mathrm{C}$ for 24 hours.

Analysis of silver nanoparticles

UV-Visible spectra analysis

Brown color appearance of the colloidal solution confirmed the AgNPs formation. Further, UV-Vis absorbance spectra were tested between the wavelength range of $300-550 \mathrm{~nm}$. Systronics double beam UV-Vis Spectrophotometer: 2201 was used for analysis and double distilled water was used in reference cuvette.

X-ray diffraction (XRD) analysis

The dried AgNPs powder was analyzed by XRD on Powder X-Ray Diffractometer (Bruker D8-Advance) at $40 \mathrm{kv}$ voltage with monochromic $\mathrm{Cu}-\mathrm{Ka}$ radiation $(\lambda=$ $1.5406 \AA$ ) at $2 \theta$ angle. The scanning region of XRD was $20^{\circ}-80^{\circ}$. The domain size of nanoparticle crystal was calculated by Scherer formula.

$\mathrm{D}=0.94 \lambda / \beta \operatorname{Cos} \Theta$

Field Emission Scanning Electron Microscope (FESEM) analysis

FE-SEM analysis was done to determine the size and shape of nanoparticles. Carl Zeiss Ultra Plus FieldEmission Scanning Electron Microscope was used at the voltage of $15 \mathrm{kV}$ in a high vacuum to reach the highest magnification.

Energy dispersive X-ray (EDX) Analysis

EDX analysis was done to analyze the abundance of silver element. Carl Zeiss Ultra Plus Field-Emission Scanning Electron Microscope with EDX attachment was used for EDX analysis.

\section{Antimicrobial activity}

Antimicrobial susceptibility of AgNPs was analyzed by disc diffusion method. $50 \mu \mathrm{g} / \mathrm{ml}$ concentration of powdered AgNPs was prepared in double-distilled water. Muller Hinton agar (MHA) medium was used to prepare bacterial culture lawn containing AgNPs discs at $37^{\circ} \mathrm{C}$ for 24 hours. Ciprofloxacin $(5 \mathrm{mcg}$ ) and distilled water discs were used as positive and negative control respectively.

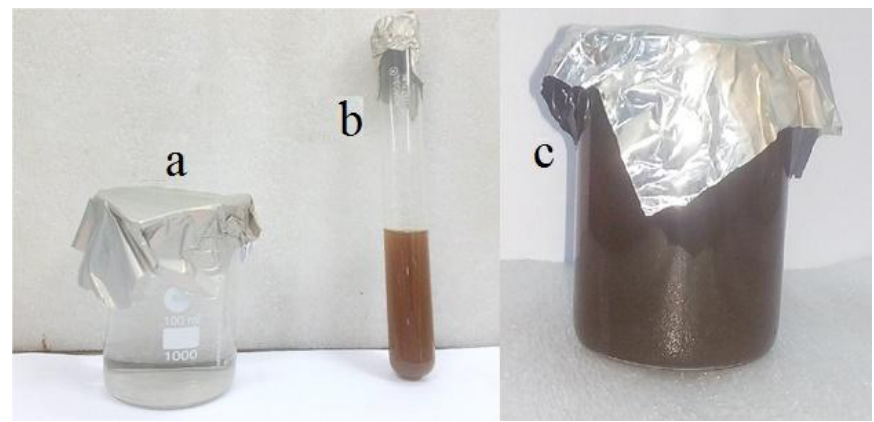

Fig. 1: Photographs of a: $\mathrm{AgNO}_{3}$, b: Plant extract, and c: AgNPs Solution

\section{RESULTS AND DISCUSSION}

UV-Visible spectra analysis

The formation of the AgNPs indicated by the color change of the silver nitrate solution after adding the plant extract, during this reduction process colorless solution changed into brown color (Figure 1). UVVisible absorption spectra of AgNPs solutions were analyzed between $300-550 \mathrm{~nm}$ and the surface plasmon resonance (SPR) bands were observed strongly between 401-415 nm (Figure 2). The range of SPR bands confirms the abundance of spherical AgNPs. The SPR peak of AgNPs was found between the $350-550 \mathrm{~nm}$ 
wavelengths. ${ }^{[4]}$ The SPR peak of biogenic (Urtica dioica Linn. leaves) synthesized silver nanoparticles at 414 nm, was similar to our observation. [10]

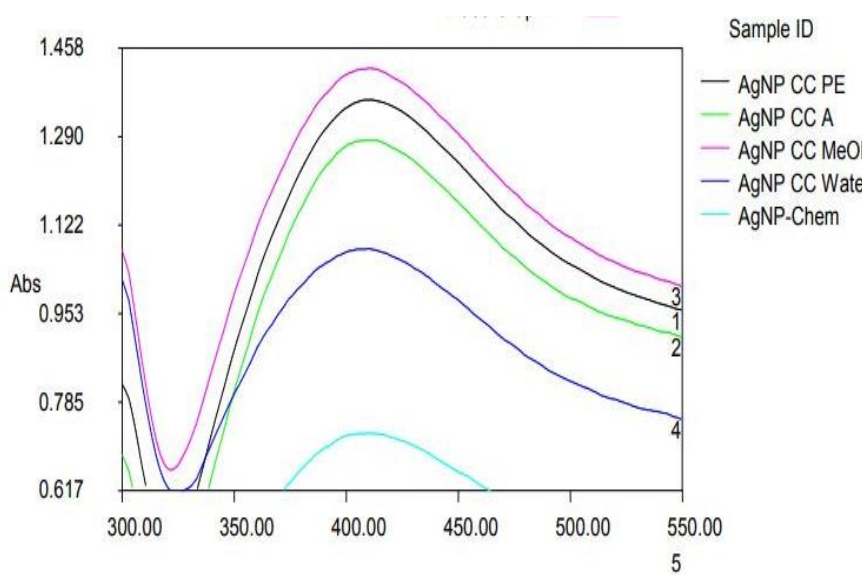

Fig. 2: UV-Vis spectra of synthesized AgNPs by C. cristata leaves extract of different solvent (PE: Petroleum ether, A: Acetone, MeOH: Methanol and Water) and chemically synthesized AgNPs (AgNPs-Chem)

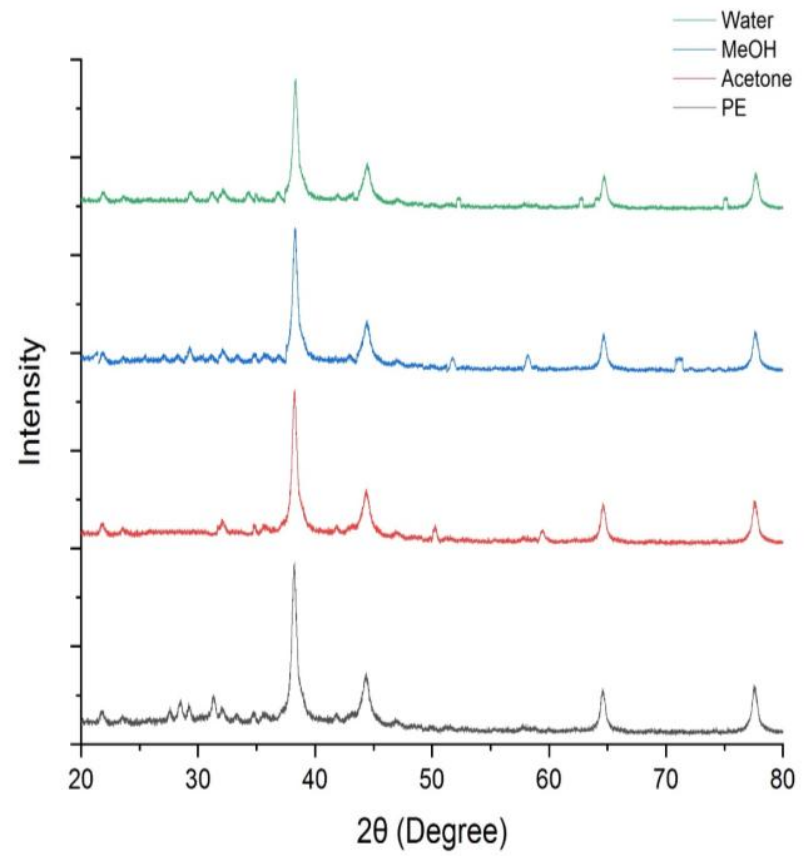

Fig. 3: XRD pattern of synthesized AgNPs by C. cristata leaves extract of different solvent (PE: Petroleum ether, Acetone, $\mathrm{MeOH}$ Methanol and Water)

\section{XRD Analysis}

The crystalline properties of AgNPs were analyzed by XRD pattern (Figure 3). Four main peaks were observed in each XRD pattern of biogenic (C. cristata leaves extract of petroleum ether, acetone, methanol, and water) synthesized AgNPs. The diffraction peaks at $38.23^{\circ}(111), 44.37^{\circ}(200), 64.64^{\circ}(220)$ and $77.65^{\circ}$ (311) for petroleum ether; $38.25^{\circ}(111), 44.39^{\circ}(200), 64.66^{\circ}(220)$, and $77.68^{\circ}$ (311) for acetone; $38.24^{\circ}(111), 44.36^{\circ}(200)$, $64.67^{\circ}(220)$ and $77.59^{\circ}$ (311) for methanol; $38.28^{\circ}(111)$, $44.40^{\circ}(200), 64.69^{\circ}(220)$ and $77.62^{\circ}(311)$ for water were observed and suggested that all crystals had FCC structure which is further confirmed by JCPDS file no. 84-0713, 04-0783 and 87-0720. The mean crystal size was $13.08,13.25,13.25$ and $13.00 \mathrm{~nm}$ respectively. Similar diffraction peaks were observed by Ghozali et al [4], Philip [11], and Shankar et al. [5]

FE-SEM analysis

Crystal shape, size, and morphology of synthesized AgNPs were analyzed by FE-SEM at 25000X. Individual and aggregates of AgNPs were observed in FE-SEM images (Figure 4). All particles were spherical in shape while aggregated AgNPs had no well-defined shape. The size of AgNPs in images was 10-60nm also observed by Vanaja \& Annadurai. [12]
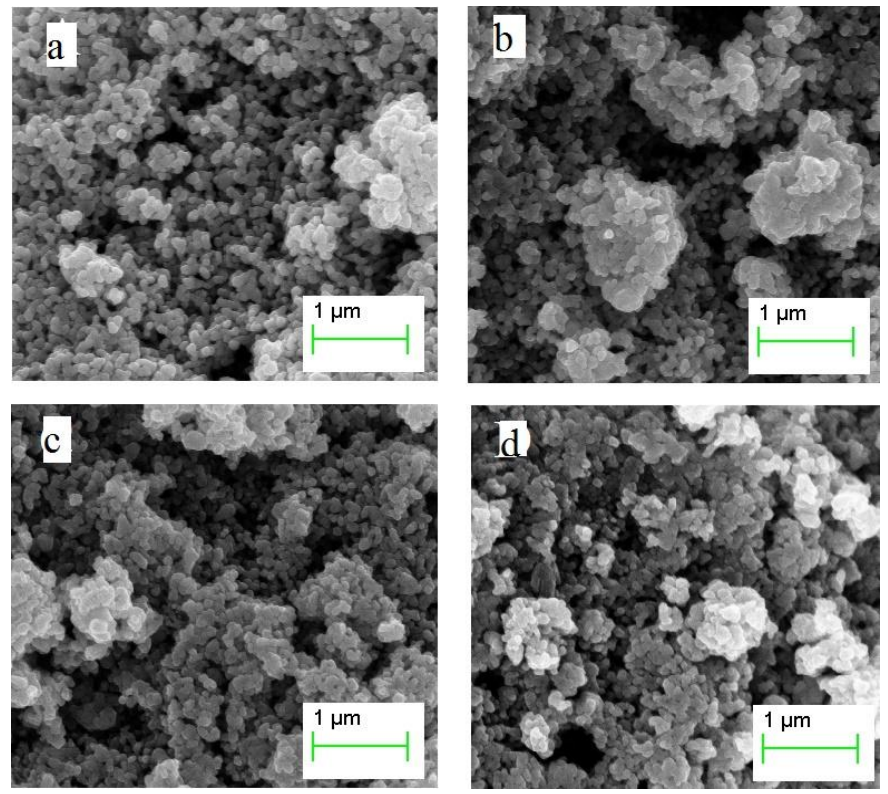

Fig. 4: FE-SEM images synthesized AgNPs by $C$. cristata leaves extract of different solvent (a: Petroleum ether, b: Acetone, c: Methanol and d: Water)

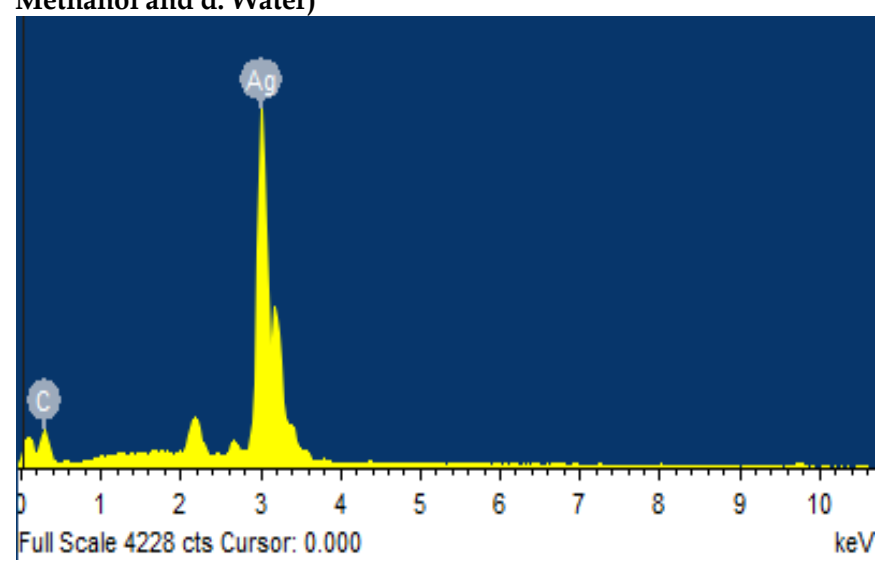

Fig. 5: EDX pattern of AgNPs

\section{EDX Analysis}

A strong peak of silver metal was observed at $3 \mathrm{keV}$ which confirms the silver nanoparticle synthesis while a weak peak of oxygen was also observed on EDX (Figure 5). No other peaks were observed in EDX which confirm the highest purity of synthesized AgNPs.

Antimicrobial activity

Antimicrobial activity of biogenic synthesized nanoparticles was analyzed by the disc diffusion method; the inhibition zone was calculated in diameter against otorhinolaryngological isolates namely IS-1, IS3 (Alcaligens faecalis), IS-4, IS-6, IS-7 (Staphylococcus sciuri), IS-8 and IS-10 (Bacillus subtilis). These 7 isolated 
bacteria were tested against biogenic synthesized and chemically synthesized AgNPs; ciprofloxacin $(5 \mathrm{mcg}$ ) and distilled water were used as positive and negative control respectively. Biogenic synthesized nanoparticles were more toxic against IS-3, IS-4, IS-6, and IS-7 as compared to ciprofloxacin while all isolates showed more zone of inhibition for biogenic synthesized nanoparticles as compares to chemically synthesized nanoparticles. The highest zone of inhibition $(28.33 \pm$ $0.88 \mathrm{~mm}$ ) was shown by methanol leaves extract AgNPs in IS-4 while least zone of inhibition (10.67 \pm $0.88 \mathrm{~mm}$ ) was shown by petroleum ether extract AgNPs in IS-7 (Figure: 6). Almost similar observations were found by Anandalakshmi et al [13], they synthesized biogenic AgNPs of Pedalium murex leaves extract and found the highest antibacterial activity against Escherichia coli and Bacillus subtilis. While Mahitha et al [14] synthesized silver nanoparticles of Bacopa monniera whole plant extract and found antibacterial activity against Staphylococcus aureas, B. subtillis, E. coli, and Klebsiella pneumoniae.

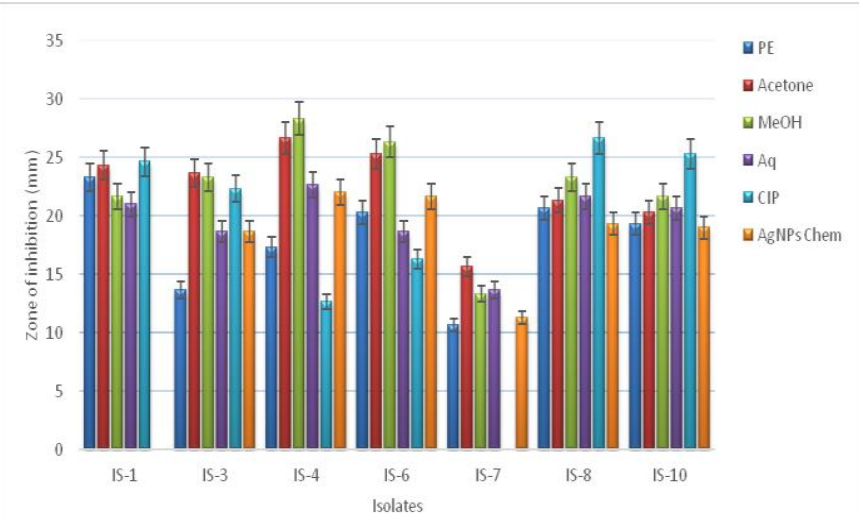

Fig. 6: Antimicrobial activity of synthesized AgNPs by C. cristata leaves extract of different solvents (PE: Petroleum ether, Acetone, MeOH: Methanol and Water), CIP: ciprofloxacin and chemically synthesized AgNPs (AgNPs-Chem)

The present work demonstrated that C. cristata leaves are capable to synthesize the biogenic AgNPs, $C$. cristata leaves extracts work like a reducing and capping agents. Characterization of AgNPs was performed by UV-Vis spectrophotometer, $\mathrm{XRD}$, FESEM, and EDX. SPR in AgNPs was confirmed by the UV-Vis absorption spectra, the abundance of silver metal in AgNPs was confirmed by EDX, FE-SEM study suggested that AgNPs had uniformly spherical shape with 10-60 nm in size range and FCC crystal structure were confirmed by XRD pattern. The biogenic synthesized AgNPs showed excellent antimicrobial activity against IS-3 (A. faecalis), IS-4, IS-6, and IS-7 (S. sciuri).

\section{ACKNOWLEDGMENTS}

The first author expresses their thankfulness to the department of Botany and Microbiology, Gurukula Kangri Vishwavidyalaya, Haridwar for providing the necessary support to carry out this research work.

\section{REFERENCES}

1. Umoren SA, Obot IB, Gasem ZM. Green synthesis and characterization of silver nanoparticles using red apple (Malus domestica) fruit extract at room temperature. Journal of Material Environmental Science 2014; 5(3):907-914.

2. Alarcon E, Griffith M, Udekwu KI. Silver nanoparticle applications: In the fabrication and design of medical and biosensing devices, Springer International Publishing, 2015.

3. Rogers KR, Bradham $\mathrm{K}$, Tolaymat $\mathrm{T}$ et al. Alterations in physical state of silver nanoparticles exposed to synthetic human stomach fluid. Science of the Total Environment 2012; 420:334-339.

4. Ghozali SZ, Vuanghao L, Ahmad NH. Biosynthesis and characterization of silver nanoparticles using Catharanthus roseus leaf extract and its proliferative effects on cancer cell lines. Journal of Nanomedicine \& Nanotechnology 2015; 6(4):1-6.

5. Shankar SS, Ahmad A, Sastry M. Geranium leaf assisted biosynthesis of silver nanoparticles. Biotechnology Progress 2003; 19(6):1627-1631.

6. Sarvamangala D, Kondala K, Murthy USN, Rao BN, Sharma GVR, Satyanarayana R. Biogenic synthesis of AGNP's using Pomelo fruit - Characterization and antimicrobial activity against Gram+ Ve and Gram- Ve bacteria. International Journal of Pharmaceutical Sciences Review and Research 2013; 19(2):30-35.

7. Danabalan $R$, Dharmalingam $S$, Kalusalingam A. Phytochemical investigation and anthelmintic activity of Celosia cristata leaf extracts. International Research Journal of Pharmacy 2012; 3(5):335-337

8. Kumar PS. Phytochemical and pharmacological overview of Celosia cristata and future perspective as potential phytotherapeutic agent. European Journal of Biomedical and Pharmaceutical Sciences 2018; 5(1):829-834

9. Surse SN, Shrivastava B, Sharma P, Sharma J, Gide PS. Pharmacognostic standardisation of whole plant of Celosia argentea var. Cristata (L). International Journal for Pharmaceutical Reaseach Scholars 2014; 3(3):387-392.

10. Jyoti K, Baunthiyal M, Singh A. Characterization of silver nanoparticles synthesized using Urtica dioica Linn. leaves and their synergistic effects with antibiotics. Journal of Radiation Research and Applied Sciences 2016; 9(3):217-227.

11. Philip D. Biosynthesis of Au, Ag and Au-Ag nanoparticles using edible mushroom extract. Spectrochimica Acta Part A: Molecular and Biomolecular Spectroscopy 2009; 73(2):374381.

12. Vanaja M, Annadurai G. Coleus aromaticus leaf extract mediated synthesis of silver nanoparticles and its bactericidal activity. Applied Nanoscience 2013; 3:217-223.

13. Anandalakshmi K, Venugobal J, Ramasamy V. Characterization of silver nanoparticles by green synthesis method using Pedalium murex leaf extract and their antibacterial activity. Applied Nanoscience 2016; 6(3):399-408.

14. Mahitha B, Raju BDP, Dillip GR et al. Biosynthesis, characterization and antimicrobial studies of AgNPs extract from Bacopa monniera whole plant. Digest Journal of Nanomaterials and Biostructures 2011; 6(2):587-594. 